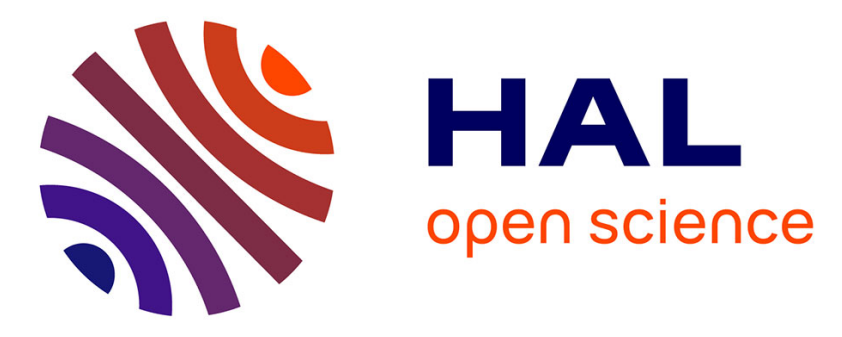

\title{
Photoluminescence polarization and piezoelectric properties of InAs/InP quantum rod-nanowires
}

Roman Anufriev, Nicolas Chauvin, Jean-Baptiste Barakat, Hammadi

Khmissi, Khalid Naji, Gilles Patriarche, Xavier Letartre, Michel Gendry, Catherine Bru-Chevallier

\section{To cite this version:}

Roman Anufriev, Nicolas Chauvin, Jean-Baptiste Barakat, Hammadi Khmissi, Khalid Naji, et al.. Photoluminescence polarization and piezoelectric properties of InAs/InP quantum rod-nanowires. CSW2014, May 2014, Montpellier, France. hal-02071832

\section{HAL Id: hal-02071832 https://hal.science/hal-02071832}

Submitted on 18 Mar 2019

HAL is a multi-disciplinary open access archive for the deposit and dissemination of scientific research documents, whether they are published or not. The documents may come from teaching and research institutions in France or abroad, or from public or private research centers.
L'archive ouverte pluridisciplinaire HAL, est destinée au dépôt et à la diffusion de documents scientifiques de niveau recherche, publiés ou non, émanant des établissements d'enseignement et de recherche français ou étrangers, des laboratoires publics ou privés. 


\title{
Photoluminescence polarization and piezoelectric properties of InAs/InP quantum rod-nanowires
}

\author{
Roman Anufriev ${ }^{1}$, Nicolas Chauvin ${ }^{1}$, Jean-Baptiste Barakat ${ }^{2}$, Hammadi Khmissi ${ }^{3}$, Khalid Naji ${ }^{2}$, Gilles Patriarche ${ }^{4}$, \\ Xavier Letartre ${ }^{2}$, Michel Gendry ${ }^{2}$, Catherine Bru-Chevallier ${ }^{1}$ \\ 1: Université de Lyon, Institut des Nanotechnologies de Lyon (INL)-UMR5270-CNRS, INSA-Lyon, 7 avenue Jean Capelle, \\ 69621 Villeurbanne, France \\ 2: Université de Lyon, Institut des Nanotechnologies de Lyon (INL)-UMR5270-CNRS, Ecole Centrale de Lyon, 36 avenue Guy \\ de Collongue, 69134 Ecully, France \\ 3: Université de Monastir, Laboratoire de Micro-Optoélectronique et Nanostructures (LMON), Faculté des Sciences, Avenue de \\ l'environnement, 5019 Monastir, Tunisia \\ 4: Laboratoire de Photonique et de Nanostructures (LPN), UPR20-CNRS, route de Nozay, 91460 Marcoussis, France
}

e-mail: nicolas.chauvin@insa-lyon.fr

Purely wurtzite InAs/InP quantum rod nanowires (QRod-NWs) emitting at $1.55 \mu \mathrm{m}$ have been successfully grown on silicon substrates by VLS assisted molecular beam epitaxy. Microphotoluminescence studies of single QRod-NWs reveal a highly linearly polarized emission parallel to the nanowires axis. This very high degree of linear polarization $(>0.9)$ can be explained by the photonic nature of the NW structure. Moreover, these QRod-NWs reveal a broad peak with an asymmetric lineshape at 10K. From experimental and theoretical studies, we conclude that this feature is a consequence of a piezoelectric field induced by the strained InAs QRod.

\section{INTRODUCTION}

The growth of nanowires (NWs) using the Vapor-LiquidSolid (VLS) method is well adapted to integrate III-V semiconductors on silicon $(\mathrm{Si})$ giving to the NW technology a great potential in optoelectronics and photonics on $\mathrm{Si}$ substrates. One original feature of III-V semiconductor NW growth is the possibility to obtain heterostructures with a wurtzite $(\mathrm{Wz})$ crystallographic phase instead of the more usual zinc-blende phase. In the present work, we report on the experimental observation of a piezoelectric field and a strong polarization anisotropy in the photoluminescence spectra of Wz InAs/InP quantum rod (QRod) NWs.

\section{GROWTH CONDITIONS AND SAMPLE PREPARATION}

The Wz InAs/InP QRod-NWs were grown by VLS assisted solid source MBE on a (111) oriented Si substrate [1]. The obtained structure consists of an InP NW with an InAs insertion, called QRod due to its elongated shape (Figure 1.a). The NWs have a $80-100 \mathrm{~nm}$ diameter and a $1.5-2 \mu \mathrm{m}$ length. The diameter and the length of the InAs QRods are measured in the $8-11 \mathrm{~nm}$ range and the $20-100 \mathrm{~nm}$ range, correspondingly. To study the NWs by means of low temperature micro-photoluminescence (micro-PL), the NWs were ultrasonically removed from the as-grown substrate and transferred onto a TEM copper grid, served as a host-substrate.
The sample was mounted in a He-flow cryostat and cooled to $10 \mathrm{~K}$. The excitation was provided by a continuous wave $\mathrm{He}$ Ne laser beam $(632.8 \mathrm{~nm})$ focused by a microscope objective to a spot size of $\approx 4 \mu \mathrm{m}$.

\section{POLARIZATION RESOLVED PHOTOLUMINESCENCE}

Polarized micro-PL studies have been performed on single QRod-NWs at 10K and room temperature. A strong anisotropy is observed: nearly all the PL emission is polarized parallel to the NW axis (Figure 1.b and 1.c). The degree of linear polarization (DLP) defined as $D L P=I_{/ /}-I_{\perp} / I_{/ /}+I_{\perp}$ is bigger than 0.9 and is temperature independent. This result is in contradiction with the fact that the ground state of the bulk Wz InAs should emit light perpendicular to the NW axis. This result could be explained either by a heavy-hole light-hole mixing [2] and/or by the photonic nature of the NW which inhibits the dipole oriented perpendicular to the NW axis when the NW diameter is small compared to the emission wavelength [3].

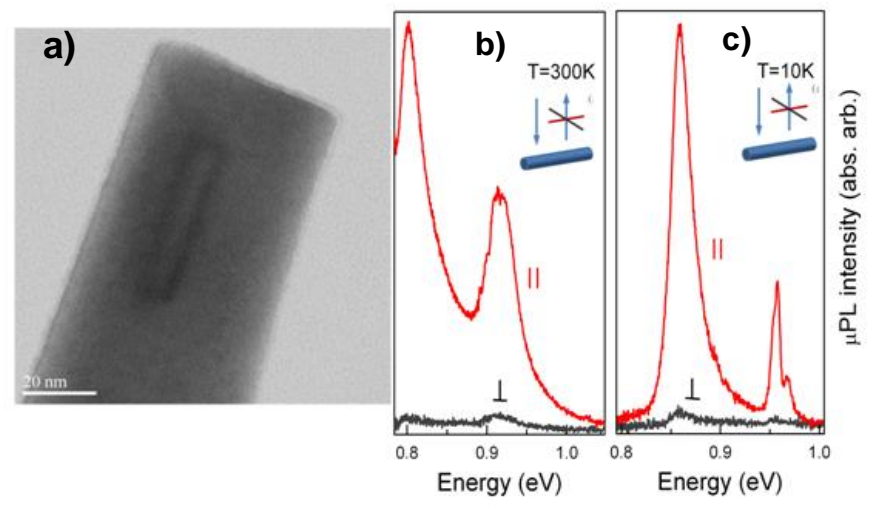

Figure 1. a) TEM picture of an InAs/InP QRod-NW. Polarization resolved PL of a single QRod-NW measured at $300 \mathrm{~K}(\mathrm{~b})$ and $10 \mathrm{~K}$ (c).

To investigate this last hypothesis, a second sample has been grown with the same growth conditions except a bigger 
InP growth time during the last step of the growth to increase the NW diameter. With this sample, we were able to investigate the polarization emission for NWs with diameters in the 100-300 $\mathrm{nm}$ range. With increasing NW diameter, a decrease of the QRod emission anisotropy is observed and the DLP is equal to 0.45 for a $300 \mathrm{~nm} \mathrm{NW}$ diameter. A strong decrease of the DLP is also observed for the $\mathrm{Wz}$ InP peak: the DLP, which is close to zero for thin NWs, reaches -0.6 for NW diameters of $300 \mathrm{~nm}$. This negative DLP means that the emission is mainly perpendicular to the NW axis, in agreement with the theory, and confirms that the DLP measured in thin NW is impacted by the photonic properties of the NW.

\section{PIEZOELECTRIC EFFECTS IN QUANTUM ROD-NANOWIRES}

The micro-PL emission of single QRod-NW ground states has been investigated, at $10 \mathrm{~K}$, as a function of the excitation power and an unusual behavior has been observed. Being broad at low excitation power, the peak is blue-shifting and narrowing as the excitation power is increased up to $0.2 \mathrm{~mW}$ (Figure 2.a).

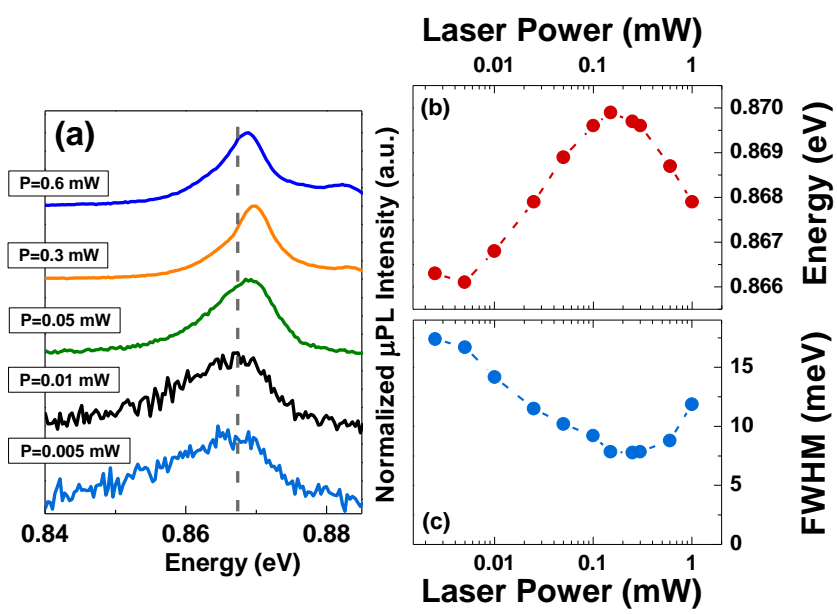

Figure 2. $10 \mathrm{~K} \mu$-PL spectra (a), emission energy (b) and linewidth (c) of a single QRod-NW as a function of the excitation power.

The blueshift and narrowing of the PL emission with the excitation power increase are typically related to an electric field screening and explained in terms of quantum confinement Stark effect (QCSE) [4]. Indeed, at low excitation power, the emission energy is decreased and the linewidth is broad due to the carrier separation induced by the electric field. However, with a laser power increase, the electric field can be screened by photogenerated carriers, weakening the QCSE and leading to an emission blueshift (Figure 2.b) and a narrowing (Fig 2.c) of the PL emission [5]. As the excitation power is reaching 0.2 $\mathrm{mW}$, the electric field is completely screened, and the following increase of the power is leading to the more usual redshift and broadening. In the present case, due to the lattice mismatch between InAs and InP materials, the $\mathrm{Wz}$ InAs QRods are strained, so a strain-induced piezoelectric polarization inducing an electric field may indeed be expected.
To investigate this phenomenon, observed at $10 \mathrm{~K}$, the same excitation power dependence studies have been performed as a function of the temperature. A blueshift and a narrowing are observed up to $50 \mathrm{~K}$ but their respective magnitudes decrease with the temperature increase and, starting from $70 \mathrm{~K}$, no clear shift or narrowing is observed. This transition, observed between $50-70 \mathrm{~K}$, is equivalent to a thermal energy $\mathrm{kT}=4-6$ $\mathrm{meV}$, value in the order of magnitude of the blueshift observed at $10 \mathrm{~K}$. This means that the carriers are thermally activated and can overcome the energy of the piezoelectric field.

In order to fully understand the piezoelectric phenomenon taking place in the QRods and the role of the QRod geometry, the calculation of the expected strain and piezoelectric field inside these Wz QRod-NWs has been performed using the 3D nano device simulator "Nextnano" [6]. An averaged electric field of $40 \mathrm{kV} / \mathrm{cm}$ is obtained in the case of a QRod of $40 \mathrm{~nm}$ in length and $8 \mathrm{~nm}$ in diameter. This piezoelectric field is strongly related to the diameter and length of the QRod. This could explain why the phenomena observed in Figure 2 were not observed for all the QRods of the sample.

In conclusion, we report on the evidences of the straininduced piezoelectric field in Wz InAs/InP QRod-NWs. This electric field caused by the lattice mismatch between the InAs and InP results in a QCSE and, as a consequence, affects the optical properties of the structure. Moreover, these QRods revealed a very strong polarization anisotropy (DLP>0.9) which is a consequence of the photonic nature of InP NWs.

\section{ACKNOWLEDGMENT}

This work has been supported by the Agence Nationale pour la Recherche project INSCOOP (ANR-11-NANO-012) and by the LABEX iMUST (ANR-10-LABX-0064) of Université de Lyon, within the program "Investissements d'Avenir" (ANR-11-IDEX-0007) operated by the French National Research Agency (ANR). Authors are gratefully acknowledging facilities and technological staffs from Nanolyon platform.

\section{REFERENCES}

[1] R. Anufriev, et al., "Polarization properties of single and ensembles of InAs/InP quantum rod nanowires emitting in the telecom wavelengths," J. Appl. Phys. 113, 193101 (2013).

[2] J. Bleuse, et al., "Inhibition, enhancement, and control of spontaneous emission in photonic nanowires," Phys. Rev. Letters 106, 103601 (2011).

[3] M. Zieliński, Phys. Rev. B 88, 115424 (2013).

[4] J. Lähnemann, O. Brandt, C. Pfüller, T. Flissikowski, U. Jahn, E. Luna, M. Hanke, M. Knelangen, A. Trampert, and H. T. Grahn, "Coexistence of quantum-confined Stark effect and localized states in an (In,Ga)N/GaN nanowire heterostructure," Phys. Rev. B 84, 155303 (2011).

[5] X.-Q. Liu, X.-L. Wang, M. Ogura, T. Guillet, V. Voliotis, and R. Grousson, "Modification of optical properties by strain-induced piezoelectric effects in ultrahigh-quality V-groove AlGaAs/GaAs single quantum wire," Appl. Phys. Lett. 80, 1894 (2002).

[6] T. Zibold, "Semiconductor based quantum information devices: Theory and simulations," PhD Thesis, Technische Universität München, 2007 